\title{
Estatus, solidaridad y representación social de las variedades de la lengua española entre hispanohablantes de Santiago de Chile*
}

\author{
Darío Rojas**
}

\begin{abstract}
Resumen
A través de una encuesta de actitudes lingüísticas, pedimos a 400 hispanohablantes santiaguinos asociar un conjunto de características extralingüísticas con distintas variedades geopolíticas del español. Los sujetos asocian el español de Chile con características relativas a la solidaridad, mientras que asocian las relativas al estatus con las variedades que funcionan como modelo normativo (español de España o de Perú). Consideramos que esto revela una representación social del español influenciada por la ideología de la lengua estándar y por la construcción histórica, a nivel lingüístico-ideológico, de una relación jerárquica entre las variedades del español.
\end{abstract}

Palabras clave: actitudes lingüísticas, representaciones sociales, estatus, solidaridad, variedades del español.

\section{Status, solidarity and social representation of varieties of Spanish among Spanish-speaking subjects from Santiago de Chile}

\begin{abstract}
By means of a linguistic attitudes survey, we asked 400 Spanish-speaking subjects from Santiago (Chile) to associate a number of non-linguistic features to geo-political varieties of Spanish. Chilean Spanish was associated with features related with solidarity, while features related with status were associated with varieties that play a role as a normative model (Castilian Spanish and Peruvian Spanish). We consider this as revealing a social representation of Spanish influenced by the ideology of standard language and by the historical linguistic-ideological construction of a hierarchical relation between the varieties of Spanish.
\end{abstract}

Key words: linguistic attitudes, social representations, status, solidarity, varieties of Spanish.

Recibido: 10-01-2013 Aceptado: 03-09-2013

* $\quad$ Esta investigación se enmarca en el proyecto NFR-193742, Linguistic Identity and Attitudes in Spanish-speaking Latin America - LIAS (Identidad y actitudes lingüísticas en Hispanoamérica LIAS), financiado por el Consejo Noruego de Investigación (Research Council of Norway).

** Chileno, Magíster en Lingüística, Universidad de Chile, Departamento de Lingüística, Universidad de Chile, Santiago, Chile, darioroj@u.uchile.cl 


\section{Introducción}

El conocimiento de las actitudes lingüísticas de los hablantes de una comunidad resulta fundamental para comprender cómo mediante el lenguaje se configuran y se difunden ideologías, representaciones sociales e identidades, sean estas individuales o grupales. En pocas palabras, el estudio de las actitudes lingüísticas ocupa un lugar central en la comprensión de la relación entre lenguaje y sociedad, preocupación que les compete de manera principal a la sociolingüística y la sociología del lenguaje (Garrett, 2001).

En el marco de la ejecución del capítulo chileno (enfocado en la capital, Santiago de Chile) del proyecto Linguistic Identity and Attitudes in Spanish-speaking Latin America (LIAS), que se propuso indagar en las actitudes lingüísticas de los hispanohablantes de 21 países, nos planteamos, entre otras, la siguiente pregunta: ¿qué características asocian los santiaguinos a la forma de hablar el español de cada uno de los países hispanohablantes? Y en relación con estas características, ¿cuál es la representación social que tienen del conjunto de estas formas de hablar?

Intentamos responder a esta pregunta aplicando un cuestionario a una muestra de 400 santiaguinos. En particular, utilizamos las respuestas a uno de los ítems de este cuestionario, en que mencionamos a los encuestados una serie de características extralingüísticas y les pedimos que indicaran a la forma de hablar español de qué país o países ellos asociaban dichas características. Interpretamos estas asociaciones como indicadoras de actitudes lingüísticas concernientes a las dimensiones valorativas del estatus y la solidaridad, y luego las analizamos como reveladoras de una representación social determinada acerca del lenguaje. En el siguiente apartado expondremos los conceptos y antecedentes pertinentes, para a continuación presentar y discutir los resultados del cuestionario.

\section{Actitudes lingüísticas, tipos de valoración del lenguaje y el caso del español de Chile}

El concepto de actitud lingüística ha resultado ser uno de los que poseen mayor importancia para entender diversas dimensiones de la interfaz lenguaje-sociedad. Los estudios sobre actitudes lingüísticas normalmente se adscriben a la sicología social del lenguaje, pero 
la sociolingüística ha mostrado un interés de igual o mayor fuerza por estos fenómenos, primeramente, porque la evaluación social de variantes lingüísticas constituye un tópico central y muy productivo en la investigación sociolingüística, pues articula la noción de competencia comunicativa (Chambers, 2003). Además, la sociolingüística ha reconocido en las actitudes lingüísticas la clave para responder a dos de las interrogantes básicas relativas al cambio lingüístico: el problema de la evaluación, principalmente, y el problema de la actuación, de manera complementaria (Tuten \& Tejedo-Herrero, 2011). La revitalización y mantenimiento de lenguas, por otra parte, depende, entre diversos factores, de las actitudes que los usuarios y los grupos dominantes tengan hacia estos códigos comunicativos (Pauwels, 2004). Asimismo, los estudiosos de la interacción comunicativa han hallado en este concepto una valiosísima herramienta interpretativa (Giles \& Billings, 2004). En suma, el estudio de las actitudes lingüísticas en la actualidad resulta de interés transversal para variados campos de estudio dentro de las disciplinas que deben enfrentarse con problemas en que los aspectos sociales afectan al uso del lenguaje.

El concepto de actitud proviene de la sicología social, ámbito en que se define como una tendencia sicológica expresada mediante la evaluación de una entidad particular con algún grado de aprobación o desaprobación (Albarracín, Zanna, Johnson \& Kumkale, 2005). Lo específico de una actitud lingüística es que la entidad particular evaluada corresponde a un rasgo lingüístico o una variedad lingüística completa. Usualmente las actitudes son concebidas en términos de una estructura tripartita: creencias (componente cognitivo), emociones (componente afectivo) y conductas (componente conductual) (Bizer, 2004), elementos cuya discreción ha sido comprobada empíricamente por diversos estudios (Maio, Esses, Arnold \& Johnson, 2004; Maio, Olson, Bernard \& Luke, 2006; Trafimow \& Sheeran, 2004). El elemento cognitivo de las actitudes resulta especialmente interesante debido a que los sistemas de creencias normalmente motivan y subyacen a actitudes de diverso signo hacia determinados objetos de tipo lingüístico. Estos sistemas de creencias han sido estudiados como teorías populares del lenguaje (Niedzielski \& Preston, 2003), ideologías lingüísticas (Kroskrity, 2010) o culturas lingüísticas (Schiffman, 1996), tomando en préstamo de la sicología social, de la antropología y de la sociología las nociones de modelo cultural, representación social e ideología y aplicándolas al estudio de las ideas 
acerca del lenguaje. Un hallazgo importante de estos estudios es que las actitudes y representaciones sociales acerca del lenguaje reflejan procesos históricos y condiciones culturales de orden más general, y que muchas veces no tienen que ver con las características estrictamente lingüísticas (pronunciación, vocabulario, etc.) de las variedades o lenguas evaluadas por los hablantes (Edwards, 2011). De este modo, el estudio de estas actitudes y representaciones constituye un observatorio privilegiado para los estudios sociales, en general.

En las investigaciones sobre actitudes lingüísticas, se ha descubierto que la valoración de las variedades lingüísticas normalmente se hace en torno a dos grandes dimensiones valorativas: el estatus y la solidaridad (Edwards, 2011). Se relacionan con el estatus conceptos como la corrección, que juega un papel central en culturas lingüísticas como la norteamericana (Preston, 2002) o la hispánica (Coseriu, 1990) y que está estrechamente vinculado con la dimensión cognitiva de las actitudes (creencias, ideologías lingüísticas, etc.), mientras que se relacionan con la solidaridad conceptos como el del agrado. Son varios los estudios de actitudes lingüísticas que han identificado dimensiones valorativas recurrentes y se han propuesto agruparlas. Zahn y Hopper (1985) consideran que las tres principales macrodimensiones de valoración del lenguaje son la superioridad (educación, riqueza, etc.), el atractivo social (amigabilidad, honestidad, etc.) y el dinamismo (energía, entusiasmo, etc.). Edwards (2011) indica, basado en una amplia cantidad de estudios sobre este asunto, que las principales macrodimensiones serían la competencia (inteligencia, etc.), integridad personal (confiabilidad, etc.) y el atractivo social (amigabilidad, sentido del humor, etc.); no obstante, investigaciones recientes sugieren que podrían establecerse simplemente dos grandes categorías: estatus social, más o menos equivalente a la competencia, y solidaridad, que aunaría integridad y atractivo social. Bayard, Weatherall, Gallois y Pittam (2001), por su parte, agrupan las valoraciones identificadas en su estudio de acuerdo con las siguientes esferas: poder (control, autoridad, asertividad, etc.), competencia (inteligencia, educación, confiabilidad, etc.), solidaridad (amigabilidad, calidez, alegría, etc.) y estatus (ingreso, clase social, etc.). En un estudio realizado desde la aproximación teórico-metodológica de la lingüística popular, Garrett, Williams y Evans (2005) clasifican las palabras clave de carácter evaluativo expresadas por sus informantes de acuerdo con cuatro categorías: rasgos lingüísticos (descripciones no técnicas de 
diversas características fónicas, gramaticales, léxicas o discursivas), afecto (agradable, divertido, etc.), estatus y normas sociales (inteligente, correcto, etc.) y asociaciones culturales (por ejemplo, "McDonalds" para el inglés de los Estados Unidos). En el ámbito hispánico, Blas Arroyo (1995) considera como principales las siguientes macrodimensiones: atractivo personal (bondad, humildad, lealtad, etc.), atractivo social (modernidad, alegría, etc.) y estatus socioeconómico (corrección, riqueza, inteligencia, cultura, etc.). González Martínez (2008) añade, a las identificadas por Blas Arroyo, la macrodimensión de la cercanía del sistema (claridad y familiaridad).

A pesar de las clasificaciones minuciosas que puedan hacerse de los tipos de valoraciones, sobresalen las dos macrodimensiones que mencionamos al comienzo: el estatus y la solidaridad. Además, estas dos macrodimensiones han revelado ser claves para entender las diferentes valoraciones que reciben algunas variedades geográficas de determinadas lenguas. Diversos estudios realizados en otros ámbitos lingüísticos (Edwards, 2011) sugieren que normalmente las variedades que son valoradas positivamente en la dimensión del estatus (por ejemplo, los estándares suprarregionales) reciben una evaluación negativa en la dimensión de la solidaridad, mientras que las variedades peor valoradas en cuanto a estatus (por ejemplo, los dialectos no estándares) reciben una evaluación positiva en cuanto a afecto y solidaridad. Edwards y Jacobsen (1987), por otra parte, han concluido que las variedades que tienen el carácter de estándar regional (por ejemplo, el inglés de Nueva Escocia continental en Canadá) reciben valoraciones igualmente positivas en ambos tipos de dimensiones, pues, precisamente, son a la vez estándar y regionales, con lo cual pueden recibir incluso mejor valoración que los estándares suprarregionales.

En cuanto a las actitudes de los santiaguinos hacia las variedades geográficas del español, el estudio de Rojas (2012a) logró identificar a qué países hispanohablantes los santiaguinos atribuyen mayor o menor corrección idiomática. Los países a los que atribuyeron mayor corrección en su uso del español son Perú y España, mientras que al español de Chile, su propia variedad, le atribuyeron el mayor grado de incorrección. En el panorama general, el español de España resultó ser la variedad mejor valorada, debido a que, por un lado, se ajusta al perfil lingüístico del español correcto ideal (a diferencia del español chileno), y, por otro, no se encuentra asociado a un grupo carente de prestigio social (a diferencia 
de la variedad peruana). Junto con estos dos factores, actúa a favor del español peninsular la historia ideológico-lingüística de la comunidad a la que pertenecen los sujetos encuestados, en la que han primado las actitudes propeninsulares y negativas hacia la variedad chilena y las americanas en general. Rojas (2012b) ilustra en detalle el perfil lingüístico del modelo ideal de lengua española que tienen los santiaguinos, perfil que incluye detalles fónicos (consonantismo conservador, ritmo pausado, etc.) y léxicos (ausencia de modismos y groserías, riqueza de vocabulario, etc.), principalmente.

Los antecedentes expuestos nos permiten plantear la hipótesis de que los santiaguinos asociarán el español de Chile, su variedad materna, que históricamente ha ocupado una posición subordinada desde el punto de vista normativo-ideológico, con características pertenecientes a la esfera de la solidaridad, mientras que asociarán el español de España y Perú, históricamente prestigiosas, con características circunscritas a la esfera del estatus.

\section{Metodología}

\subsection{Sujetos participantes en el estudio}

La muestra de sujetos encuestados se compone de 400 individuos, distribuidos en grupos sociodemográficos ${ }^{1}$ según se muestra en el Cuadro $\mathrm{N}^{\mathrm{o}} 1$ :

1 La representación de cada uno de los estratos y grupos es proporcional a la que tienen en el universo de la población de Chile, según el censo nacional del año 2002 (www.ine.cl). Las proporciones son las siguientes: estrato alto $=11 \%$, estrato medio $=44,5 \%$, estrato bajo $=44,5 \%$; $20-34$ años $=36 \%, 35-54$ años $=40,5 \%, 55$ años o más $=23,5 \%$; hombres $=48 \%$, mujeres $=52 \%$. Para la determinación de los estratos socioeconómicos empleamos los datos de ICCOM (Instituto Consultor en Comercialización y Mercado Limitada), en su versión del año 2007 (tablas disponibles en www.iccom.cl). Las equivalencias y proporciones empleadas en nuestra investigación son las siguientes: estrato alto $=\mathrm{ABC} 1$; estrato medio $=\mathrm{C} 2+\mathrm{C} 3$; estrato bajo $=\mathrm{D}+\mathrm{E}$. ICCOM clasifica a los sujetos de acuerdo con los siguientes criterios: barrio, tipo de vivienda, nivel educacional, profesión y actividad del jefe de hogar, ingreso familiar y posesiones. 
Cuadro $\mathrm{N}^{\mathrm{o}} 1$

Distribución de los sujetos de la muestra según estrato socioeconómico (bajo, medio, alto), grupo etario (20-34, 35-54, 55 o + años) y sexo (hombre, mujer)

\begin{tabular}{lccccccc}
\hline & \multicolumn{2}{c}{$\mathbf{2 0 - 3 4}$} & \multicolumn{2}{c}{$35-54$} & \multicolumn{2}{c}{55 o + } \\
\hline & Hombre & Mujer & Hombre & Mujer & Hombre & Mujer \\
\hline Bajo & 31 & 34 & 35 & 38 & 20 & 22 \\
\hline Medio & 31 & 34 & 35 & 38 & 20 & 22 \\
\hline Alto & 7 & 7 & 8 & 8 & 5 & 5 \\
\hline & & & & & & $\mathrm{n}=400$
\end{tabular}

Los encuestados viven en el núcleo urbano del Gran Santiago ${ }^{2}$ desde hace 20 años o más (la mayor parte lo han hecho toda su vida). Aunque no asignamos una cuota de acuerdo con la representatividad demográfica de cada comuna, la mayoría proviene de un grupo de comunas que resultan prototípicas de cada estrato social. Así, por ejemplo, la mayor parte de los individuos de estrato alto proceden de Vitacura, Las Condes y Lo Barnechea, mientras que la mayor parte de los de estrato bajo proceden de La Pintana, Cerro Navia, San Ramón y Quilicura³ ${ }^{3}$

\subsection{Instrumento de recolección de datos y aplicación}

Para medir las actitudes lingüísticas de los sujetos utilizamos el método directo. En este enfoque metodológico a los encuestados se les pregunta directamente por sus preferencias, opiniones, evaluaciones, etc., sobre el lenguaje, es decir, la indagación se fundamenta en la expresión manifiesta de actitudes (Garrett, 2010). En la actualidad, gozan de mayor prestigio entre los investigadores aproximaciones de tipo indirecto, tales como la técnica de pares disfrazados o las tareas de evaluación de hablantes, en las que el diseño metodológico está orientado a ocultar a los sujetos el objeto

2 Consideramos 37 comunas: Cerrillos, Cerro Navia, Conchalí, El Bosque, Estación Central, Huechuraba, Independencia, La Cisterna, La Florida, La Granja, La Pintana, La Reina, Las Condes, Lo Barnechea, Lo Espejo, Lo Prado, Macul, Maipú, Nuñoa, Pedro Aguirre Cerda, Peñalolén, Providencia, Pudahuel, Puente Alto, Quilicura, Quinta Normal, Recoleta, Renca, San Bernardo, San Joaquín, San Miguel, San Ramón, Santiago y Vitacura. Excluimos solamente Padre Hurtado, Pirque y San José de Maipo por pertenecer más bien a áreas semirrurales y no tener relaciones muy estrechas con el resto de la capital.

3 En esta correlación nuevamente seguimos los datos de ICCOM. 
de estudio y poder llegar, así, al nivel "subconsciente" de las opiniones y valoraciones, que se vería menos afectado por la tendencia a responder de acuerdo con ideas recibidas respecto de qué es socialmente adecuado o de acuerdo con lo que creen que el encuestador espera que respondan. Sin embargo, algunos estudiosos (Garrett, Coupland \& Williams, 2003) han abogado recientemente por programas integrales de investigación, que combinen técnicas directas e indirectas para llegar a conocer en su total complejidad las actitudes lingüísticas de los hablantes. Por otro lado, a partir de la década de los 90 ha ido ganando popularidad la convicción de que la aproximación directa permite obtener información mucho más rica cualitativamente en comparación con las técnicas indirectas (véase, por ejemplo, Niedzielski \& Preston, 2003).

El instrumento utilizado consiste en un cuestionario de 40 preguntas. Este fue aplicado a los sujetos por tres colaboradoras, licenciadas con especialización en Lingüística o profesoras de Lenguaje, entre marzo y noviembre del 2010. Las colaboradoras encuestaron a los individuos en sus lugares de trabajo, en sus hogares o en lugares públicos, poniendo por escrito las respuestas en el formulario, con total fidelidad a las palabras de cada encuestado. La aplicación de cada encuesta demoró 20 minutos en promedio.

En particular, en este estudio utilizamos las respuestas a uno de los ítems de este cuestionario, en que las colaboradoras mencionaron en voz alta a los encuestados once características extralingüísticas, en este mismo orden: cariño, enojo, tecnología, elegancia, vulgaridad, sentido del humor, bajos recursos económicos, altos recursos económicos, confianza en el trato, respeto y autoridad. Tras mencionar cada característica, las colaboradoras les pidieron a los encuestados que dijeran con la forma de hablar español de qué país o países ellos asociaban esta característica. Una vez finalizada la recolección de datos, las respuestas fueron ingresadas a un formulario electrónico (de SurveyXact) desarrollado especialmente para el proyecto.

\subsection{Análisis}

Nuestro análisis se basó en el agrupamiento de las características extralingüísticas de acuerdo con las macrodimensiones valorativas del estatus y la solidaridad. Así, consideramos como relativas al estatus las siguientes características: enojo, tecnología, elegancia, altos recursos económicos, respeto y autoridad. En cambio, consideramos relacionadas 
con la solidaridad las siguientes propiedades: cariño, vulgaridad, sentido del humor, bajos recursos económicos y confianza en el trato. Contabilizamos la cantidad de menciones que obtuvo cada país en cada característica y luego se determinaron los porcentajes relativos. Para cada característica, consideramos pertinente determinar al habla de qué país era asociada con mayor frecuencia y si había alguna relación estrecha entre el habla de un determinado país y los grupos de características (estatus/solidaridad).

\section{Presentación y discusión de resultados}

\subsection{Resultados}

En el Cuadro $\mathrm{N}^{\circ} 2$ presentamos los porcentajes de menciones que obtuvo el habla de cada país al preguntársele a los encuestados con qué variedad del español asociaban las características indicadas en cada una de las columnas. En el costado izquierdo agrupamos las características relativas al estatus, y en el lado derecho las relativas a la solidaridad. Para cada característica, resaltamos en gris y con negrita el país cuya habla tiene el porcentaje más alto, y solo con negrita los que ocupan el segundo lugar. Dejamos en blanco los casos en que no hubo menciones para ese país.

Cuadro $\mathrm{N}^{\circ} 2$

Asociación de características con variedades del español (porcentajes)

\begin{tabular}{|c|c|c|c|c|c|c|c|c|c|c|}
\hline \multicolumn{5}{|c|}{ Estatus } & \multicolumn{6}{|c|}{ Solidaridad } \\
\hline$\stackrel{\circ}{\stackrel{0}{2}}$ & 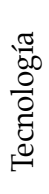 & 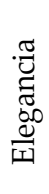 & 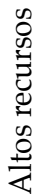 & 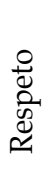 & 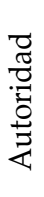 & 光 & 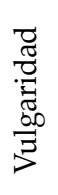 & 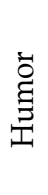 & 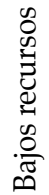 & Uే \\
\hline
\end{tabular}

\begin{tabular}{lrrrrrrrrrrr}
\hline Argentina & $\mathbf{4 4}$ & 5,8 & $\mathbf{2 2}$ & $\mathbf{1 1}$ & $\mathbf{4}$ & $\mathbf{2 5}$ & $\mathbf{4}$ & $\mathbf{1 5}$ & $\mathbf{9 , 3}$ & 0,8 & $\mathbf{1 2}$ \\
\hline Belice & & & & & & & 0,3 & 0,3 & & & \\
\hline Bolivia & 4 & & 0,3 & 0,3 & 3,3 & 0,3 & $\mathbf{1 , 5}$ & $\mathbf{7 , 3}$ & 0,5 & $\mathbf{4 2}$ & 0,8 \\
\hline Chile & $\mathbf{1 5}$ & $\mathbf{2 7}$ & $\mathbf{4 , 3}$ & $\mathbf{1 7}$ & $\mathbf{1 5 , 8}$ & $\mathbf{1 4 , 3}$ & $\mathbf{2 8}$ & $\mathbf{4 1}$ & $\mathbf{5 5}$ & 8 & $\mathbf{2 8}$ \\
\hline Colombia & 1,3 & 0,3 & $\mathbf{7 , 8}$ & $\mathbf{1 , 3}$ & $\mathbf{8 , 3}$ & $\mathbf{1 , 3}$ & $\mathbf{1 7}$ & 2 & 3,3 & 2,8 & 10,5 \\
\hline C. Rica & & & 0,8 & 0,3 & 0,3 & & 1,5 & 0,5 & 1 & 0,3 & 1 \\
\hline
\end{tabular}




\begin{tabular}{|c|c|c|c|c|c|c|c|c|c|c|c|}
\hline Cuba & 1 & 1,5 & 1 & & 3 & 4 & 7,3 & 1,5 & 6,8 & 2 & 10 \\
\hline Ecuador & 0,3 & & 1,5 & 0,5 & 3 & & 2 & & 0,3 & 0,5 & 1 \\
\hline El Salvador & & & & 0,3 & & & 0,3 & & 0,8 & & \\
\hline EE. UU. & 0,5 & 4,8 & & 3,8 & & 1 & & 0,3 & & & \\
\hline España & 13 & 18,5 & 38 & 32 & 16 & 30 & 4,3 & 1 & 1,3 & 0,3 & 3,5 \\
\hline Guatemala & & & 0,3 & 0,3 & & & & & 1 & 0,3 & \\
\hline Honduras & & & & 0,3 & & & 0,8 & & 1 & & \\
\hline México & 1,8 & 7,8 & 0,8 & 8,3 & 2,8 & 2,8 & 7,5 & 2,3 & 10,3 & 0,5 & 4,3 \\
\hline Nicaragua & & & & 0,3 & 0,5 & & & & & & \\
\hline Panamá & & & & 0,3 & & & 0,5 & & & 0,5 & 0,3 \\
\hline Paraguay & 0,3 & 0,3 & 0,3 & & 0,5 & & 1 & 0,3 & 0,5 & 0,5 & 0,5 \\
\hline Perú & 5,3 & 0 & 7,3 & 0,5 & 26 & 1 & 9,5 & 8 & 1,5 & 22 & 5,8 \\
\hline Puerto Rico & & 0,5 & 0,3 & & & 2 & 7,5 & 3,3 & 0,8 & 3,8 & \\
\hline R. Dominic. & & & & & & 0,3 & 0,5 & 0,8 & & 0,8 & \\
\hline Uruguay & 0,8 & 0,3 & 1,8 & 2 & 3 & 0,3 & 1 & 0,5 & & 2,3 & \\
\hline Venezuela & 5 & 1 & 1,5 & 2,5 & 2,3 & 4,5 & 6,5 & 3,5 & 3,5 & 1,5 & 6,5 \\
\hline Ninguno & 6,8 & 28 & 11,5 & 16,3 & 8,3 & 10,5 & 5,8 & 4,8 & 1,8 & 13 & 6,5 \\
\hline No sabe & 1 & 4,8 & 1 & 4,5 & 2,5 & 4,8 & 1,3 & 3,8 & 1,5 & 3 & 3 \\
\hline
\end{tabular}

Fuente: elaboración propia

Nuestros resultados revelan que, en conformidad con nuestra hipótesis, el habla propia de los participantes (Chile) es mejor valorada en dimensiones pertenecientes al ámbito de la solidaridad (cariño, humor, confianza, vulgaridad) mientras que en las dimensiones relativas al estatus (elegancia, autoridad, altos recursos) tiene mayor preferencia el español de España.

Esta tendencia se puede apreciar de mejor manera en los Gráficos 1 y 2: 
Gráfico $\mathrm{N}^{\mathrm{o}} 1$

Asociación de características con el español de Chile (porcentajes)

\section{Chile}

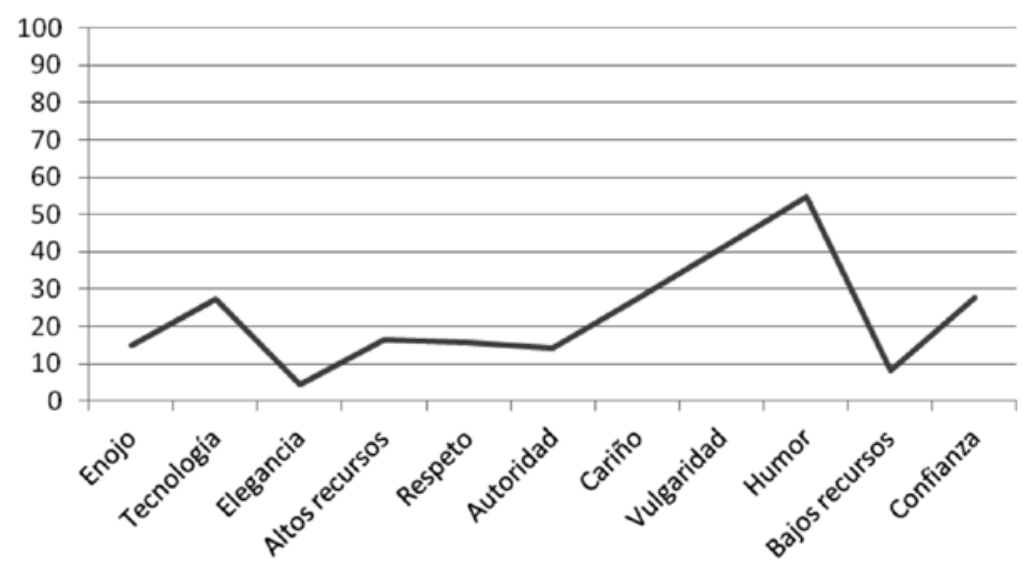

Fuente: elaboración propia

Gráfico $\mathrm{N}^{\mathrm{o}} 2$

Asociación de características con el español de España (porcentajes)

\section{España}

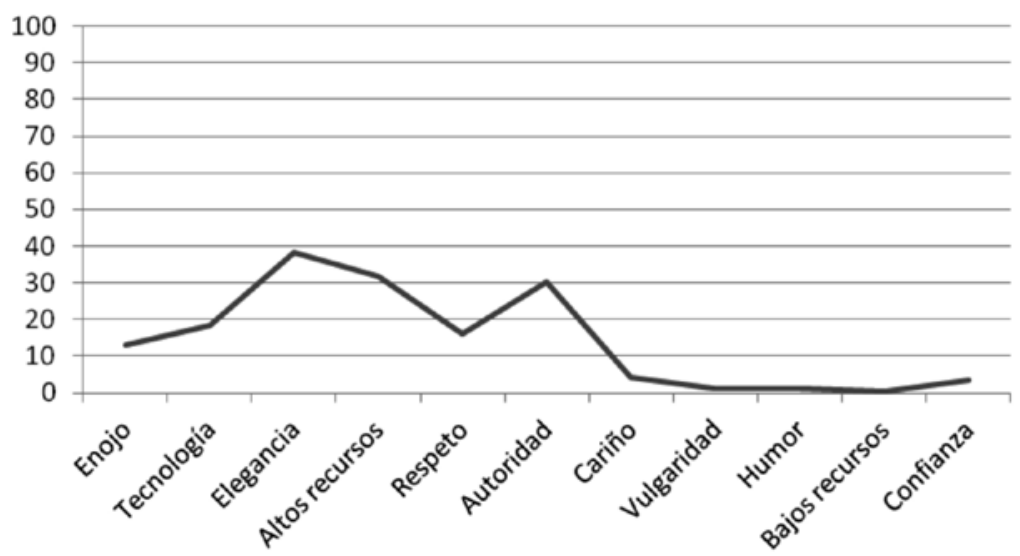

Fuente: elaboración propia 
En ambos gráficos las características relativas al estatus se encuentran agrupadas en la mitad izquierda, mientras que a la derecha están agrupadas las características relacionadas con la solidaridad. Estos gráficos muestran que la valoración del español de Chile se orienta hacia la derecha, es decir, hacia la solidaridad, mientras que la del español de España se inclina hacia la izquierda, esto es, hacia el estatus.

En ninguno de los otros países se da una tendencia como esta de manera clara. Cabe destacar, por último, que los datos presentados no muestran diferencias relevantes entre los distintos grupos sociodemográficos considerados en la muestra de participantes (estrato socioeconómico, edad y sexo).

\subsection{Discusión}

La tendencia que acabamos de presentar es congruente con lo hallado por Rojas (2012a) en cuanto a que el español de España es uno de los mejor valorados en términos de corrección idiomática. Cabe mencionar que el otro país cuya forma de hablar español es muy bien valorada por los santiaguinos en términos de corrección, Perú, recibe la preferencia más alta en la dimensión del respeto, que también pertenece al ámbito del estatus. Existe congruencia, entonces, entre la atribución de un alto grado de corrección idiomática y la asociación a características de la dimensión del estatus. La congruencia también se da en la otra dirección: los resultados de Rojas (2012a) muestran que el habla de Chile fue percibida como la más incorrecta, y en esta ocasión se la asocia a características no relacionadas con el estatus sino con la solidaridad.

En conclusión, se cumple el patrón que se puede comprobar en estudios de otros ámbitos lingüísticos (cf. sección 2): las variedades que funcionan o alguna vez han funcionado como estándares suprarregionales (español de España, español de Perú) reciben la mejor valoración en la dimensión del estatus, mientras que las variedades carentes de prestigio reciben una evaluación positiva únicamente en la dimensión de la solidaridad (español de Chile). Para comprender adecuadamente estas diferentes valoraciones, sin embargo, es necesario examinar los antecedentes histórico-lingüísticos de esta situación.

Desde las reflexiones americanas decimonónicas acerca de la diversificación dialectal del español se puede observar que la valoración de las variedades geográficas de la lengua española en América se ha 
hecho teniendo como modelo idiomático al español de la metrópoli peninsular, de acuerdo con lo cual se ha concebido que las variedades que muestren mayor concordancia lingüística con dicha variedad (en los niveles fonológico-fonético, gramatical y léxico; véase por ejemplo Rojas (2012b) para un perfil lingüístico del modelo de lengua española que manejan los santiaguinos) son de mejor calidad. Durante la época colonial americana, es posible rastrear diversos juicios que dan cuenta del prestigio que se le asignaba, además de al español de la metrópoli peninsular, al español del los virreinatos de México y Lima, así como al español hablado en las zonas altas de Colombia (Guitarte, 1991; Quesada, 2002). Estas variedades, precisamente, son las que se han conformado históricamente como variedades de consonantismo conservador (Moreno Fernández, 2009), lo cual parece ser un rasgo sumamente saliente en las percepciones lingüísticas de los hispanohablantes. En estas variedades, por ejemplo, el sonido /s/ en posición implosiva no se aspira ni elide, sino que se articula plenamente; este rasgo es constantemente nombrado por los santiaguinos al momento de justificar sus atribuciones de corrección, en términos de que la aspiración o elisión es incorrecta. El perfil fónico conservador o innovador de las variedades americanas del español, según ha mostrado Germán de Granda (1994), depende en términos históricos de si las entidades geopolíticas que les servían de contexto tenían el carácter de zonas centrales, es decir, con gran poder económico y alto grado de desarrollo cultural y social (por ejemplo, México, Lima) o zonas periféricas, de pocos recursos económicos y escaso desarrollo sociocultural (por ejemplo, Paraguay), o bien se ubicaban en un punto intermedio entre lo central y lo periférico (por ejemplo, Chile). En resumen, el español de Chile y otras variedades que divergen estructuralmente del modelo peninsular metropolitano han sido consideradas tradicionalmente, por sus propios usuarios, como formas deslegitimadas y periféricas en relación con la norma ejemplar peninsular, y han sido asociadas, por tanto, a la incorrección idiomática (Coseriu, 1990: 73-74), creando entonces una situación de inseguridad lingüística.

La percepción de las variedades geográficas de la lengua española por parte de los chilenos también se ha visto muy marcada por la construcción ideológico-discursiva del estatus diferencial asignado a cada una de ellas. El español metropolitano peninsular ha desempeñado el papel de modelo para la conciencia lingüística de los hispanohablantes 
americanos desde la época de la Colonia y al menos hasta la época de la Independencia (Guitarte, 1991). Los debates lingüístico-ideológicos de comienzos de la historia del Chile independiente desembocaron en el triunfo de los unionistas (Quesada, 2002), quienes tenían como modelo principal el español peninsular. Un representante de esta clase de opiniones es Andrés Bello, quien, a pesar de su defensa de las particularidades lingüísticas americanas, pensaba que los chilenos (y americanos) debían aprender a distinguir, en la pronunciación, entre eses y zetas, como los españoles ${ }^{4}$. Asimismo, tienen el mismo cariz las opiniones de varios diccionaristas chilenos del s. XIX, como Rodríguez (1979 [1875]), quien constantemente contrapone los usos chilenos censurables a los usos corrientes en España, que, en su opinión, tenían mayor legitimidad. En particular, las opiniones de Bello en materias idiomáticas, como es sabido, tuvieron repercusiones importantes tanto en Chile como en el resto de Hispanoamérica (Moré, 2002). La frase con que Rodríguez (1979 [1875]: vii) abre su Diccionario: “La incorrección con que en Chile se habla y escribe la lengua española es un mal tan generalmente reconocido como deplorado", refleja, por otra parte, el tratamiento de que ha sido objeto históricamente la variedad chilena por parte de sus mismos usuarios. Afirmaciones de este tipo pueden encontrarse entre muchos gramáticos y diccionaristas de los siglos XIX y XX, y pueden rastrearse asimismo en el discurso público actual acerca del español chileno. Conforman un amplio corpus de ejemplos de la inseguridad que caracteriza y ha caracterizado la autoimagen lingüística de los chilenos.

Las circunstancias que acabamos de reseñar explican, en nuestra opinión, la asociación del español de España, principalmente, y de Perú con el estatus, y la vinculación del español de Chile con la solidaridad. La representación social o modelo cultural del lenguaje que se deja ver detrás de nuestros resultados se corresponde grosso modo con el ideal platónico descrito por Niedzielski y Preston (2003) a partir de datos relativos al inglés de los Estados Unidos. Según estos autores, los no especialistas creen en la lengua como un ideal platónico que tiene existencia más allá del uso de los hablantes. La actuación lingüística apropiada, para estos

4 “Los que se cuidan de evitar todo resabio de vulgarismo en su pronunciación [...], si aspiran a una pronunciación más esmerada, distinguirán también la s de la z o c [...]; de manera que suenen de diverso modo la casa que habitamos y la caza de los animales silvestres; la cima a que se sube y la sima a que se desciende" (Bello, 1940 [1833-1834]: 66). Para otros ejemplos que revelan que Bello juzgaba el habla de España superior a la de Chile, véase Moré (2002). 
sujetos, debe corresponder con ese ideal de lengua, y toda conducta que se aleje de ella es considerada como error. En esta categoría de error cabe no solamente la variación lingüística casual y asistemática (por ejemplo, la causada por distracción o ebriedad del hablante), sino que también variedades sociales o geográficas completas, tales como, en el caso del inglés estadounidense, los dialectos regionales o el inglés vernáculo afroamericano. Las variedades regionales, en consecuencia, no pueden poseer la propiedad de la corrección, pues esta se halla solamente en el uso congruente con el ideal lingüístico. La misma imagen parece aplicarse al caso del español; sin embargo, cabe hacer la observación de que en esta percepción la atribución de corrección probablemente no sea efectuada en forma categórica sino prototípica: una variedad geográfica del español será más susceptible de ser considerada como correcta en la medida en que comparta rasgos con el modelo ideal de lengua.

Esta visión de la lengua es compatible con lo que Milroy (2001) denomina la ideología de la lengua estándar y lo que Del Valle y GabrielStheeman (2002) llaman la ideología monoglósica. Ambos sistemas de creencias conciben el lenguaje como una entidad idealmente uniforme, articulada en torno a un modelo que funciona como medida de calidad de toda conducta lingüística. La configuración centro-periferia que adopta el lenguaje en estos modelos culturales impone una asimetría jerárquica en la valoración de las variedades de la lengua española: aquellas que se encuentren más cerca del centro resultarán mejor valoradas, mientras que las más lejanas a ese centro serán peor valoradas. Ya que el concepto clave que caracteriza al centro de este modelo es el de la corrección, que se asocia normalmente con educación, competencia, etc., es natural que las variedades que se encuentren más cerca del centro sean mejor valoradas en las dimensiones del estatus. Por otro lado, para que una variedad situada en la periferia sea objeto de valoraciones positivas, basta con que actúe la tendencia al favoritismo intragrupal (Myers, 2010: 326-328) y prime, en la conciencia de los sujetos, la identificación afectiva con la variedad propia por sobre la consideración de su carácter periférico. Lo primero es lo que sucede con el español de España, y lo segundo es lo que ocurre con el español de Chile. 


\section{Conclusiones}

A través de las asociaciones que los santiaguinos hacen entre algunas características extralingüísticas y las formas de hablar español de diversos países se puede vislumbrar una diferencia valorativa de acuerdo con si prima la percepción de estatus o el sentimiento de solidaridad. El español de España se encuentra asociado al estatus, mientras que el español de Chile se vincula preferentemente con la solidaridad. Esto se relaciona estrechamente con la asimetría jerárquica (en términos de centro-periferia) que imponen la ideología de la lengua estándar y la historia lingüístico-ideológica chilena entre las variedades que ejercen o han ejercido el papel de modelo suprarregional (por ejemplo, el español de España) y aquellas que no lo han hecho (por ejemplo, el español de Chile). Se puede comprobar, entonces, que la representación social que los sujetos tienen acerca de su propia lengua influye en las actitudes que muestran hacia sus distintas variedades geográficas.

Nuestros resultados e interpretación constituyen, por supuesto, una aproximación preliminar a este problema. Hemos trabajado con un conjunto reducido de características valorativas que hasta ahora se encuentran entre las más usadas en los estudios de actitudes lingüísticas, pero que sin duda convendría enriquecer cuantitativa y cualitativamente. Por otra parte, hemos aplicado un enfoque metodológico directo, que convendría complementar con una metodología de tipo indirecto, como la técnica de pares disfrazados. La continuación de la indagación en estas direcciones, creemos, contribuirá a dilucidar con mayor claridad nuestra interrogante inicial. 


\section{Referencias bibliográficas}

Albarracín, D.; Zanna, M. P., Johnson; B. T. \& Kumkale, G. T. (2005). Attitudes: Introduction and Scope. En D. Albarracín, B. T. Johnson \& M. P. Zanna (eds.), The Handbook of Attitudes (pp. 3-19). Mahwah/London: Lawrence Erlbaum Associates.

Bayard, D.; Weatherall A.; Gallois, C. \& Pittam, J. (2001). Pax Americana?: Accent attitudinal evaluations in New Zealand, Australia and America. Journal of Sociolinguistics 5. 22-49.

Bello, A. (1940 [1833-34]). Advertencias sobre el uso de la lengua castellana, dirigidas a los padres de familia, profesores de los colegios y maestros de escuela. En El español en Chile, Biblioteca de Dialectología Hispanoamericana, tomo VI. Buenos Aires: Instituto de Filología de la Universidad de Buenos Aires. 5077.

Bizer, G. (2004). Attitudes. En Ch. Spielberger (ed.), Encyclopedia of Applied Psychology, vol. 1. London: Elsevier Academic Press. 245-249.

Blas Arroyo, J. L. (1995). De nuevo el español y el catalán, juntos y en contraste. Estudio de actitudes lingüísticas. Sintagma 7. 29-41.

(1999). Las actitudes hacia la variación intradialectal en la sociolingüística hispánica. Estudios Filológicos 34. 47-72.

Chambers, J. K. (2003). Studying Language Variation: An Informal Epistemology. En J. K., Chambers, P. Trudgill \& N. Schilling-Estes (eds), The Handbook of Language Variation and Change. Malden: Blackwell. 3-14.

Coseriu, E. (1990). El español de América y la unidad del idioma. En Actas del I Simposio de Filología Iberoamericana. Zaragoza: Pórtico. 43-75. 
Del Valle, J. \& Gabriel-Stheeman, L. (2002). Nationalism, hispanismo and monoglossic culture. En J. del Valle \& L. GabrielStheeman (eds.), The Battle over Spanish between 1800 and 2000. Language ideologies and Hispanic intellectuals. London/New York: Routledge. 1-13.

Edwards, J. (2011). Challenges in the Social Life of Language. London: Palgrave Macmillan.

Edwards, J. \& Jacobsen, M. (1987). Standard and Regional Standard Speech: Distinctions and Similarities. Language in Society 16 (3). 369-379.

Garrett, P. (2001). Language Attitudes and Sociolinguistics. Journal of Sociolinguistics 5 (4). 626-631.

__ (2010). Attitudes to Language. Cambridge: Cambridge University Press.

Garrett, P.; Coupland, N. \& Williams, A. (2003). Investigating Language Attitudes: social meanings of dialect, ethnicity and performance. Cardiff: University of Wales Press.

Garrett, P.; Williams, A. \& Evans, B. (2005). Attitudinal data from New Zealand, Australia, the USA and the UK about each other's Englishes: Recent changes or consequences of methodology? Multilingua 24. 211-235.

Giles, H. \& Billings, A. (2004). Assessing Language Attitudes: Speaker Evaluation Studies. En A. Davies \& C. Elder (eds.), The Handbook of Applied Linguistics. Malden: Blackwell. 187-209.

González Martínez, J. (2008). Metodología para el estudio de las actitudes lingüísticas. En I. Olza Moreno, M. Casado Velarde \& R. González Ruiz (eds.), Actas del XXXVII Simposio Internacional de la Sociedad Española de Lingüística (SEL). Pamplona: Servicio de Publicaciones de la Universidad de Navarra. 229-238.

Granda, G. de (1994). Formación y evolución del español de América. Época colonial. En Español de América, español de África y hablas criollas hispánicas. Cambios, contactos y contextos. Madrid: Gredos. 49-92. 
Guitarte, G. (1991). Del español de España al español de veinte naciones: la integración de América al concepto de Lengua Española. En Actas del III Congreso Internacional sobre el español de América 69-90. Valladolid: Junta de Castilla y León.

Kroskrity, P. V. (2010). Language ideologies - Evolving perspectives. En J. Jaspers, J.-O. Östman \& J. Verschueren (eds.), Society and Language Use. Amsterdam/Philadelphia: John Benjamins. 192-211.

Maio, G. R.; Esses, V.; Arnold, K. \& Olson, J. (2004). The function-structure model of attitudes. Incorporating the need for affect. En G. Haddock \& G. R. Maio (eds.), Contemporary Perspectives on the Psychology of Attitudes. Hove/ New York: Psychology Press. 9-33.

Maio, G. R.; Olson, J. M.; Bernard, M. M. \& Luke, M. A. (2006). Ideologies, Values, Attitudes, and Behavior. En J. Delamater (ed.), Handbook of Social Psychology. New York: Springer. 283-308.

Milroy, J. (2001). Language ideologies and the consequences of standardization. Journal of Sociolinguistics 5 (4). 530555.

Moré, B. (2002). The ideological construction of an empirical base. Selection and elaboration in Andrés Bello's grammar. En J. del Valle \& L. Gabriel-Stheeman (eds.), The Battle over Spanish between 1800 and 2000. Language ideologies and Hispanic intellectuals. London/New York: Routledge. 42-63.

Moreno Fernández, F. (2009). La lengua española en su geografía. Madrid: Arco/Libros.

Myers, D. (2010). Social Psychology, 10.a ed. New York: McGraw-Hill.

Niedzielski, N. A. \& Preston, D. R. (2003). Folk Linguistics. Berlin/New York: Mouton de Gruyter.

Pauwels, A. (2004). Language Maintenance. En A. Davies \& C. Elder (eds.), The Handbook of Applied Linguistics. Malden: Blackwell. 719-737. 
Preston, D. (2002). Language with an Attitude. En J. K. Chambers, P. Trudgill \& N. Schilling-Estes (eds.), The Handbook of Language Variation and Change. Malden: Blackwell. 40-66.

Quesada, M. Á. (2002). El español de América, 2.a edic. Cartago: Editorial Tecnológica de Costa Rica.

Rodríguez, Z. (1979 [1875]). Diccionario de chilenismos, edición facsimilar. Valparaíso: Editorial Universitaria de Valparaíso.

Rojas, D. (2012a). Corrección idiomática atribuida al español de los países hispanohablantes por sujetos de Santiago de Chile. Revista de Lingüística Teórica y Aplicada 50 (2). 39-62.

(2012b). Actitudes lingüísticas de hispanohablantes de Santiago de Chile: creencias sobre la corrección idiomática. Onomázein 26. 69-93.

Schiffman, H. (1996). Linguistic Culture and Language Policy. London: Routledge.

Trafimow, D. \& Sheeran, P. (2004). A theory about the translation of cognition into affect and behavior. En G. Haddock \& G. R. Maio (eds.), Contemporary Perspectives on the Psychology of Attitudes. Hove/New York: Psychology Press. 57-75.

Tuten, D. \& Tejedo-Herrero, F. (2011). The relationship between historical linguistics and sociolinguistics. En M. Díaz Campos (ed.), The Handbook of Hispanic Sociolinguistics. Malden: Wiley-Blackwell. 283-302.

Zahn, C. \& Hopper, R. (1985). Measuring language attitudes: the speech evaluation instrument. Journal of Language and Social Psychology 4. 113-123. 